\title{
Towards Achieving Linear Capacity Scaling in Wireless Networks through Directed Energy Links
}

\author{
Hong Huang*, Yousef Jaradat*, Satyajayant Misra ${ }^{\dagger}$, Reza Tourani $^{\dagger}$ \\ *Klipsch School of Electrical and Computer Engineering, New Mexico State University \\ \{hhuang, yousef77\}@nmsu.edu \\ ${ }^{\dagger}$ Department of Computer Science, New Mexico State University \\ \{misra, rtourani\}@cs.nmsu.edu
}

\begin{abstract}
Large-scale multi-hop wireless networks have many important applications. However, Gupta and Kumar showed that the capacity of multi-hop wireless networks decreases as the number of nodes in the network increases. Subsequent research efforts to achieve linear capacity scaling have significant limitations such as long latency, high technical complexity, restricted traffic pattern, or infrastructure requirement. We propose to achieve close-to-linear (CTL) capacity scaling through the use of directed energy (DE) links such as laser communications links or highly directional pencil beam links in the EHF band in a hybrid network that also contains traditional omni-directional (OD) antenna links. Our approach has none of limitations mentioned earlier. We show that when the probability distribution of $\mathrm{DE}$ links follows the inverse-square law, a distributed scheme with local routing information suffice to achieve CTL capacity scaling.
\end{abstract}

Index Terms-wireless networks, transport capacity, performance analysis and modeling.

\section{INTRODUCTION}

I $\mathrm{N}$ their seminal work [1], Gupta and Kumar showed that the per-node throughput of multi-hop wireless networks of $n$ nodes scales as $O(1 / \sqrt{n \log n})$, which diminishes as $n$ increases. This finding caused great concerns for the scalability of wireless networks. For a network to scale, linear scaling of the capacity is desirable, which means the capacity of the network grows linearly with the number of nodes, and the increase of the network size does not lead to performance degradation. Subsequent research indicated that linear capacity scaling is feasible in certain scenarios [11]-[15]. But all these scenarios have severe limitations and downsides such as unbounded delay [11], technical complexity whose feasibility is still not clear [12], [13], traffic pattern restriction that might be impractical [14], and infrastructure requirement that is unrealistic in many practical settings [15].

In this paper, we propose an approach to Close-To-Linear (CTL) capacity scaling through the use of directed energy (DE) links, such as highly directional pencil beam links in the EHF frequency band [21] or laser communications links. CTL scaling means the per-node throughput scales as $\Theta\left(1 /(\log n)^{a}\right)$, with $a$ being a constant. The factor $1 /(\log n)^{a}$ is due to the random deployment of the nodes, which is also present in the Gupta and Kumar's capacity results (where $a=1 / 2$ ). Our approach has none of the limitations associated with previous attempts to achieve linear capacity scaling.
In addition, our approach addresses another challenge in large-scale networking: the high cost of distributing global routing information. Although the capacity of large-scale wireless networks have been much studied, not much attention has been paid to how to implement practical routing protocols in large-scale wireless networks. It is unrealistic to ignore network dynamics in large-scale networks: nodes can join, leave, or move, and channels can fade. The network links are especially volatile when DE links are involved. However, distribution of global routing information incurs high cost. In a network of $n$ nodes, the overhead of a routing protocol such as the link state protocol scales as $n^{2}$, whereas the total network capacity scales as $n / \log n$ [1], which could be overwhelmed by the routing overhead as $n$ goes to infinity. We show that when the probability distribution of DE links follows the inverse-square law, global routing information is not required to achieve CTL capacity scaling using our approach.

Radio transmission using the omni-directional (OD) antennas is an immensely wasteful way of sending information in so far as energy is concerned, since only a tiny portion of the energy transmitted is collected by the receiver, with the rest being worse than waste because of the interference it causes. In contrast, with DE links, the signal-bearing power does not spread out quickly over distance, making it an excellent candidate for long-distance links. In fact, DE links are significantly more focused than traditional directionalantenna links. The range of a directed energy link can be as high as tens of kilometers [18], which is on the same order of magnitude as the diameter of a typical large deployment (e.g., battlefields or disaster areas). However, long-distance DE links are highly fragile: they require line of sight, precise alignment, and good weather conditions and they can be disrupted by the movement of nodes, rain, fog, dust, heat, natural or artificial obstructions, etc. This poses a challenging problem of how to utilize highly fragile DE links effectively.

In this paper, we show that a significant capacity increase can be obtained in a large-scale network by utilizing the DE links even when the establishments of such links are unpredictable random events. In our approach, a packet uses OD hops to randomly sample the DE links until it encounters a suitable DE link and makes a long-distance jump. So, we have a hybrid wireless network with OD links providing 
opportunities for the effective usage of DE links. Recent advances made in network science provide the evidence of the viability of our approach. The proposed hybrid network bears a strong resemblance to the classical small world phenomenon originally studied by Milgram [24], and recently followed up by Watts and Strogatz [25], and Kleinberg [19], [20], among others. Kleinberg showed that the nodes are connected by a surprisingly small number of hops ("six degrees of separation") in a large network with a mixture of short-distance and long-distance links, which mirrors a hybrid network of OD and DE links. Since the capacity in multi-hop wireless network is limited by the number of hops a packet has to travel to reach the destination [1], small number of hops translates to high capacity.

Admittedly, our approach to the CTL capacity scaling comes at the cost of extra spectrum of the DE links. But Gupta and Kumar proved that it is impossible to achieve the CTL capacity scaling without new resources or technology. Our requirement for extra spectrum of the DE links is modest: each node has a small probability, say 0.01 , to have a single DE link, and the network formed by the DE link does not have to be connected. In fact, as the network becomes larger, the opportunity for a node to establish a DE link with any other node becomes more abundant. Furthermore, our approach can effectively deal with the highly dynamic nature of DE links by utilizing DE links greedily whenever and wherever they appear and without global coordination.

\section{A. Overview of Our Results}

In the following, we provide a sketch of some of our technical findings so that the motivations and the issues can be better understood.

The necessary condition for CTL capacity scaling: In a network of $n$ nodes, let the per-node throughput (bits per second or bps) be $\lambda$, the average number of hops per connection be $h$, the link capacity be $B$ (bps), and the total number of transmissions in the network be $n_{t x}$. The fundamental constraint is that the traffic demand can not exceed capacity supply, i.e. $n \lambda h=n_{t x} B$ or $\lambda=n_{t x} B / n h$. Since $n_{t x}=O(n / \log n)$ [1], CTL capacity scaling is possibly only if $h=O\left((\log n)^{a}\right)$, which we call the necessary condition for CTL capacity scaling. Note that the existence of DE links does not change $n_{t x}$ order-wise [16].

The achievability for CTL capacity scaling under powerlaw link distribution: As in [19], we assume that the DE link distribution follows the power law. More precisely, let $f(r)$ be the probability density that a node has a DE link with another node separated with a distance $r$. Power-law link distribution means that $f(r)=C / r^{\alpha}, \alpha$ being a nonnegative constant and $C$ being the normalization constant.

It is revealing to examine the case of $\alpha=0$. In such case, the graph formed by the nodes and DE links is the random graph famously studied by Erdös and Rényi [26]. It is well known that the diameter of such random graph is $O(\log n)$ hops. For these random graphs, the necessary condition for CTL capacity scaling is satisfied. However, we will show in Section IV that even though short paths exist in the random graph $(\alpha=0)$, the probability of finding them goes to zero as $\mathrm{n}$ goes to infinity if global routing information is not available. By global routing information, we mean that a node knows about every link in the network enabling it to compute the shortest paths to every other node in the network. We show that without global routing information, CTL capacity scaling is achievable only if $\alpha=2$ exactly, even when smaller $\alpha$ implies more DE links. In other words, the presence of more long-distance DE links does not automatically guarantee high capacity.

The availability of global routing information in a largescale wireless network is unlikely for the following two reasons. 1) It is well known that the distribution of global routing information in networks is very costly, especially for largescale networks. In fact, the number of link-update messages scales as $n^{2}$, which rapidly outpaces the total capacity supply $n_{t x} B=O(n / \log n)$ as $n$ goes to infinity. 2) More importantly, even if the distribution of global routing information is feasible, the value of such information is doubtful because DE links are highly dynamic and the information about a distant link might already be outdated when the information is received.

\section{B. Contributions}

The contributions of this paper are as follows.

- We provide an approach to CTL capacity scaling through the combined use of DE and OD links. Our approach has none of the limitations of the previously proposed approaches, refer to Table I. Furthermore, our approach can effectively deal with the high dynamics of DE links.

- We show that if global routing information is not available, the CTL capacity scaling is achievable when the DE link probability distribution follows the inverse-square law with respect to the distance between two nodes, and it is not achievable if the distribution follows any other power-law distribution. Not requiring global routing information solves another challenging problem in largescale networking, i.e., the high cost of distributing routing information.

- We provide the bounds on the capacity of the hybrid wireless networks that contain both DE and OD links.

The paper is organized as follows. In Section II, we describe related work. In Section III, we state our assumptions, definitions, and background results. In Section IV, we describe the achievability of the CTL condition under different $\alpha$ values. In Section V, we investigate the per-node throughput capacity of the hybrid network. In Section VI, we synthesize the results obtained in different theorems. We conclude in Section VII.

\section{RELATED WORK}

The study of the capacity of large-scale wireless networks started in the seminal paper [1]. Many subsequent studies have followed their leads. Upper bounds to the capacity were provided in relation to geographic locations and power constraints [2]. Information theoretical analyses were provided in [3], [4], which is different from the previous models that are based on geometry or signal-to-noise ratio (SNR). The capacity of wireless networks was studied over fading channels 
TABLE I: COMPARISONS BETWEEN OUR APPROACH AND PREVIOUS APPROACHES TO ACHIEVE LINEAR OR CTL CAPACITY SCALING IN WIRELESS NETWORKS

\begin{tabular}{|c|c|c|c|c|}
\hline \multirow{2}{*}{ Approaches } & \multicolumn{4}{|c|}{ Limitations } \\
\hline & Long Delay & Complex Technology & Restricted Traffic Pattern & Infrastructure Requirement \\
\hline Node Mobility [11] & Yes & No & No & No \\
\hline Network-wide MIMO [12], [13] & No & Yes & No & No \\
\hline Local Traffic [14] & No & No & Yes & No \\
\hline Hybrid Wireless Networks with Base Stations [15] & No & No & No & Yes \\
\hline Our Approach & No & No & No & No \\
\hline
\end{tabular}

[5], in various path-loss attenuation regimes [6], [7], and in the fixed SNR regime [8]. The gap between the upper bound and the achievable capacity was closed using an analysis based on percolation theory in [9]. Capacity regimes of wireless networks with arbitrary size and densities were studied in [10].

Previous work showed that linear capacity scaling is feasible in four scenarios. The first scenario is where node mobility assists the transport of packets [11]. The downside of the proposed approach is that it can incur unbounded delays. The second scenario is where network-wide MIMO transmission is orchestrated [12], [13]. The problem is the high implementation complexity involved, whose feasibility is not yet clear. The third scenario is where traffic demand is localized [14]. The problem is that real-world traffic is often not localized. The fourth scenario is where the wireless network is augmented with a wire-line infrastructure [15]. The limitation is that there is no such infrastructure in many important application scenarios.

The most relevant previous works to ours are [16], [17], [23]. In [16], the capacity of wireless networks with directional antennas was studied. The papers results indicate that a gain of $2 \pi / \theta$ ( $\theta$ being the beam-width) in throughput per node can be achieved by using directional antennas over that of omnidirectional antennas. Such constant gain still does not make the capacity scalable. In [16], the capacity gain is achieved mostly from interference avoidance. Our results indicate that much larger gain in capacity can be obtained by range-extension than interference-avoidance. In [17], a network of $n$ nodes was considered, in which all the nodes are equipped with $\mathrm{RF}$ transceivers and a fraction of nodes (m nodes) are also equipped with FSO transceivers. The capacity of such network is given as $W_{1}(n \log n)^{-1 / 2}+W_{2}(m \log m)^{1 / 2} / n$, where $W_{1}$ and $W_{2}$ are the bandwidths of RF and FSO links. The capacity scaling is roughly $n^{-1 / 2}$, not even close to linear scaling. Such pessimistic result stems from the authors assumption that the range of FSO links is constrained to be the minimum to form a connected topology. Such assumption is unnecessary and overly conservative, and leads to pessimistic capacity scaling. In [23], the maximum throughput problem is studied under three scenarios: 1) omnidirectional transmission, 2) simple directional transmissions, and 3) complex directional transmissions, in which the sender can generate multiple beams aimed at multiple receives. The authors show that an increase of $\Theta\left((\log n)^{2}\right)$ in maximum throughput can be achieved by using complex transmissions. None of the previous work addressed the issue of the high cost of distributing global information nor the issue of handling highly dynamic DE links.

Other related works include the following. In [29], the capacity of hybrid wireless networks with omnidirectional and directional antennas was studied. This work was later extended to investigating the multicast capacity in a similar setting [31]. In [28], collaborative data dissemination in cognitive vehicular networks was studied with the focus on investigating the sensing-throughput tradeoff. In [30], social ties were exploited to enhance device-to-device communications in wireless networks.

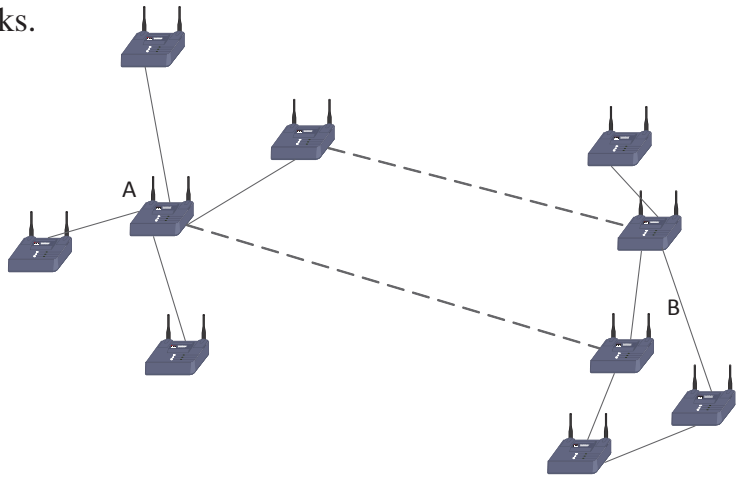

Fig. 1: A hybrid networks composed of OD (solid) and DE (dashed) links.

\section{ASSUMPTIONS, DEFINITIONS, AND BACKGROUND RESULTS}

\section{A. Assumptions}

1) Node deployment: The network has $n$ nodes uniformly randomly deployed in a disc of area $n$, which is called the extended model in the literature. In the literature there is also the dense model where nodes are deployed in a disc of unit area. Since the capacity results from the two models are basically the same, we choose to use the extended model.

2) Traffic pattern: Each node randomly selects a destination node and sends data packet at the rate of $\lambda$ bits per second.

3) Network links: The nodes can have two kinds of links: short range omni-directional (OD) wireless links and long range directed energy (DE) links, referring to Fig. 1. An OD link with capacity $B_{1}$ exists between two nodes if the distance of the nodes is no more than a threshold distance $r_{1}$. A DE link has a capacity $B_{2}$ and an average distance of $r_{2}$. The DE link probability distribution is described by the next assumption.

4) DE link distribution: Each node has a small probability $p$, say $p=0.01$, of establishing a single DE link. Let $f(r)$ be the probability density that a node has a DE link with another node separated with a distance $r$. As in [19], we assume that $f(r)=C / r^{\alpha}$, $\alpha$ being a nonnegative constant called clustering exponent. The integration of 
$f(r)$ over the network area is $p$. The model can capture a wide range of distance relationships. We also assume that the events that a node has a DE link are independent.

5) Routing information: We assume that global information about the network is not available, that is, only local information is available to the nodes. Local information for a node is defined as information about itself and its one-hop neighbors.

6) Distance measure: We assume the physical distance measure is available, which is provided by geographical routing [22].

We use the following notations: $f(n)=\Theta(g(n))$ means there exist some constants $c_{1}, c_{2}$, such that $c_{1} g(n) \leq f(n) \leq$ $c_{2} g(n)$ as $n$ goes to infinity; and $f(n)=O(g(n))$ means there exists some constant $c_{3}$, such that $f(n) \leq c_{3} g(n)$ as $n$ goes to infinity.

B. Definitions

Definition 1: We say that the per-node throughput capacity is $\lambda$ if there exists a communication scheme that can provide every node in the network with a throughput of at least $\lambda$ bits per second.

Definition 2: By global routing information, we mean the information necessary to compute the shortest paths in the network. In other words, global information is the link state information of all the links in the network, which is the input to the algorithm to compute the shortest paths such as Dijkstra's algorithm.

Definition 3: We say that the necessary condition for CTL capacity scaling is met if the average number of hops (in any combinations of OD and DE hops) that a packet travels in the network is $O\left((\log n)^{a}\right)$, where $a$ is a constant.

C. Background Results

Lemma 1 (refer to [1]): For some constant $\varepsilon$, if $r_{1}=$ $(1+\varepsilon) \sqrt{\log n / \pi}$, the probability of the network being connected (no isolated node) goes to 1 as $n$ goes to infinity. If $r_{1}=(1-\varepsilon) \sqrt{\log n / \pi}$, the probability of the network being disconnected goes to 1 as $n$ goes to infinity.

Note that throughout this work log is based on 2. To ensure the connectivity of the network, we set the radio range of OD links to be:

$$
r_{1}=(1+\varepsilon) \sqrt{\frac{\log n}{\pi}}
$$

\section{THE ACHIEVABILITY OF THE NECESSARY CONDITION FOR CTL CAPACITY SCALING}

In this section, we examine the achievability of the necessary condition for CTL capacity scaling across the full spectrum of $\alpha$ values (the exponent in link probability distribution).

\section{A. The case of $\alpha=0$}

In this subsection, we show that it is impossible to meet the necessary condition for CTL capacity scaling without global information in the Erdös-Rényi random graph $(\alpha=0)$.

Theorem 1: If $\alpha=0$ and global information is not available, the number of hops between a random pair of nodes is no smaller than $\Theta\left(n^{1 / 3} / \sqrt{\log n}\right)$ with high probability, i.e., the probability goes to 1 as $n$ goes to infinity. Therefore, the necessary condition for CTL capacity scaling is not met.
Proof: Let nodes $A$ and $B$ be a random pair of nodes. In a network of area $n$, consider a disc $D$ centered at node $A$ with a radius of $n^{1 / 3}$. With high probability $\left(1-\Theta\left(n^{-2 / 3}\right)\right)$, node $B$ is outside $D$. There are two cases: A) only OD links are used, and B) a combination of OD links and DE links are used. The case of using only DE links is not feasible, because some nodes may not have DE links or have only unsuitable DE links. In Case $\mathrm{A}$, the number of hops between $A$ and $B$ is at least $n^{1 / 3} / \sqrt{\log n}$ since the OD radio range is $\sqrt{\log n}$ (Lemma 1). In Case B, a packet uses OD hops to move to different nodes and sample different DE links. The probability of encountering a DE link to go from outside to inside $\mathrm{D}$ area is at most $P_{D}=\Theta\left(n^{-1 / 3}\right)$, given the uniform distribution of DE links. Each hop taken by the packet can be considered as an independent Bernoulli trial to get inside of D through a DE link. The number of trials, thus the number of hops, is no smaller than $1 / P_{D}=\Theta\left(n^{1 / 3}\right)$.

We know that the Erdös-Rényi random graph model $(\alpha=0)$ has a diameter of $O(\log n)$ hops. Theorem 1 indicates that without global information and only through random trials it is impossible to find the short paths of $O(\log n)$ hops. Note that a greedy approach is used in that a link is taken to reduce the distance to the destination as much as possible. But without global information, encountering DE link is a random event.

B. The case of $\alpha=2$

In this subsection, we show that the necessary condition for CTL capacity scaling can be met without global routing information when $\alpha=2$. The communication scheme, which we call Opportunistic Communication using DE links (OCDE), that meets the necessary condition for CTL capacity scaling is as follows. The packet travels from its source to its destination through $O(\log n)$ phases. In each phase, the packet does a random walk through OD links until it encounters a DE link that can halve the packets current distance to the destination (according to the assumption III-A.6, nodes know about the distances). The packet travels through the DE link and the current phase ends. We require the packet not to revisit the nodes visited in a short interval ago by keeping a short list of nodes just visited in the packet header. Revisiting a node after the short interval is equivalent to a new random sampling of DE links since DE links are highly dynamic. Thus, each phase, the distance of the packet to the destination is reduced by half. After $O(\log n)$ phases, the packet is within a few OD hops of the destination, where the packet is delivered to the destination through OD hops. In OCDE, progress is mostly made by DE links (halving the distance to destination in each phase), and OD links provide the opportunity to sample different DE links.

Of course, a couple of optimizations can be made on the above simple scheme. First, instead of doing random walks through OD links, the packet can be forwarded through an OD link to the neighbor that is the closest to the destination. Second, instead of waiting for a DE link that can reduce the distance by half, the packet can use a DE link as long as the DE hop can reduce the distance more than any potential OD hops.

We formalized our results in Theorem 2.

Theorem 2: If $\alpha=2$ and global routing information is not available, there exists, with high probability, a communica- 
tion scheme that can deliver a packet between any sourcedestination pair using $O\left((\log n)^{2}\right)$ OD hops and $O(\log n)$ DE hops. Thus, the necessary condition for CTL capacity scaling is met.

The proof of Theorem 2 is in Appendix A. Here we provide a brief outline. The packet from the source reaches its destination in $O(\log n)$ phases. In each phase, the packet's distance to the destination is reduced by half. It can be shown (see Appendix A) that if $\alpha=2$, it takes $O(\log n)$ trials (OD hops) to encounter a DE link that can reduce the distance of the packet to the destination by half. Theorem 2 is the result of the fact that there are $O(\log n)$ phases, and each phase consists of one DE hop and $O(\log n)$ OD hops.

\section{The case of $\alpha \neq 0,2$}

In this subsection, we show that it is impossible to meet the necessary condition for CTL capacity scaling without global routing information in the case of $\alpha \neq 2$.

Theorem 3: Under the condition that global information is not available, the expected number of hops, using any combinations of OD and DE links, of any communication scheme are 1) $\Theta\left(n^{(2-\alpha) / 6} / \sqrt{\log n}\right)$ hops, if $0 \leq \alpha<2$; and 2) $\Theta\left(n^{(\alpha-2) /(2 \alpha-2)} / \sqrt{\log n}\right)$ hops, if $\alpha>2$. In both cases, the necessary condition for CTL capacity scaling is not met.

The proof of Theorem 3 is in Appendix B. We provide a sketch here. For $0 \leq \alpha<2$, consider such a small disc $D$ of radius $n^{\beta}(\log n)^{1 / 4}$ centered at the destination, with the constant $\beta=((2-\alpha) / 6)<1 / 3$. A random source is initially outside $D$ with high probability. It can be shown (see Appendix B) that it takes $\Theta\left(n^{\beta} / \sqrt{\log n}\right)$ trials (OD hops) to encounter a DE link that can jump inside $D$ from the outside. Therefore, to reach the destination the total number of hops, counting both OD and DE hops, is at least $\Theta\left(n^{\beta} / \sqrt{\log n}\right)=\Theta\left(n^{(2-\alpha) / 6} / \sqrt{\log n}\right)$.

For $\alpha>2$, we denote $E$ as the event that the source node of a packet has a distance larger than $(n / 3 \pi)^{1 / 2}$ to the destination, which occurs with the probability $\operatorname{Pr}[E]=2 / 3$. Let $\beta^{\prime}=(\alpha-2) /(2 \alpha-2), \gamma=1 /(2 \alpha-2)$. It can be shown (see Appendix B) that we can bound the distance traveled by the packet in $n^{\beta^{\prime}}$ DE hops to be less than $(n / 3 \pi)^{1 / 2}$. Furthermore, we can bound the distance traveled by the packet in $n^{\beta^{\prime}} / \sqrt{\log n}$ OD hops to be also less than $(n / 3 \pi)^{1 / 2}$. Therefore, the expected number of hops $E[h]>E[h \mid E]=$ $\Theta\left(n^{(\alpha-2) /(2 \alpha-2)} / \sqrt{\log n}\right)$.

D. The Scale Invariance Property

It is interesting to note that the polylogarithmic scaling of the number of hops is achieved when $\alpha=2$, and not when $\alpha$ takes any other value. The proofs of Theorems 2 and 3 provide some insight. It turns out that there is an important property that is true only in the case of $\alpha=2$. Consider a sequence of concentric discs centered at a destination: $D_{1}, D_{2}, D_{3}, \cdots$, where $D_{1}$ covers the entire network, $D_{2}$ 's radius is half of that of $D_{1}, D_{3}$ 's radius is half of that of $D_{2}$, and so on. Suppose the packet is currently located inside $D_{i}$ but outside $D_{i+1}$ in a single trial. Let $P_{i}$ be the probability that the packet encounters a DE link that can jump inside $D_{i+1}$ in a single trial. The scale invariance property is that all $P_{i}$ 's are on the same order of magnitude with respect to $n$. The scale invariance property turns out to be the main reason why necessary condition for $C T L$ capacity scaling is met only when $\alpha=2$. For $0 \leq \alpha<2$, $P_{i}$ diminishes as $i$ gets larger, since the DE link probability distribution tends to spread out in space and does not favor smaller discs. For $\alpha>2, P_{i}$ diminishes when $i$ is small, since the DE link probability decays sharply with increasing distances. At $\alpha=2, P_{i}$ holds out steady at $O(1 / \log n)$, the inverse of which is the number of hops required to reduce the distance by half, thus achieving CTL capacity scaling.

\section{THE PER-NODE THROUGHPUT CAPACITY}

In this section, we investigate the per-node throughput capacity of the hybrid network consisting of OD and DE links. Refer to Section III-A for the definitions of the variables that will be used below.

\section{A. Upper Bound on Capacity}

In this subsection, we provide an upper bound on the pernode throughput capacity as stated in Theorem 4.

Theorem 4: For some constants $k_{2}$ and $k_{3}$, the per-node throughput capacity $\lambda$ is upper-bounded by

$$
\lambda \leq \frac{k_{2}}{\sqrt{n \log n}}+\frac{k_{3} p r_{2}}{\sqrt{n}}
$$

Note that the first term on the right hand side of (2) comes from the contributions of OD links and is the same as that in [1]; the second term comes from the contributions of the DE links, where $p$ is the probability of having a DE link for the node. The proof can be found in the technical report [27].

From Theorem 4, it is straightforward to obtain the following corollary.

Corollary 1: The necessary condition for CTL capacity scaling is $r_{2}=\Theta\left(\sqrt{n} /(\log n)^{b}\right)$, where $b$ is some constant.

Recall that $r_{2}$ is the average distance spanned by a DE link. The above necessary condition, however, is by no means sufficient, especially if global routing information is not available as is shown in Section IV.

\section{B. The CTL Capacity Scaling Can Be Achieved When $\alpha=2$}

In this subsection, we show that under the condition that global routing information is not available and $\alpha=2$, the communication scheme OCDE described in Section IV can achieve the capacity upper bound within a polylogarithmic factor.

Theorem 5: The OCDE scheme can provide a per-node throughput capacity of $\Theta\left(1 /(\log n)^{3}\right)$.

The above per-node throughput is within a polylogarithmic factor of magnitude of the capacity upper-bound in (2). Thus, the CTL capacity scaling is achieved. The proof of Theorem 5 uses the result on the expected number of hops in Theorem 2 and applies a similar approach as [1]. The details can be found in the technical report [27].

C. The CTL Capacity Scaling Can Not Be Achieved When $\alpha \neq 2$

In this subsection, we show that under the condition that global routing information is not available and $\alpha \neq 2$, it is impossible to achieve CTL capacity scaling.

Theorem 6: The per-node throughput capacity $\lambda$ is upper bounded by 1) $\Theta\left(n^{(\alpha-2) / 6} / \sqrt{\log n}\right)$, if $0 \leq \alpha<2$; and 2) 
$\Theta\left(n^{(2-\alpha) /(2 \alpha-2)} / \sqrt{\log n}\right)$, if $\alpha>2$. In both cases, the CTL capacity scaling is not achievable.

The proof of Theorem 6 can be found in the technical report [27]. From Theorem 6, we see that the capacity scaling is strictly sublinear when $\alpha \neq 2$. The network capacity achieves its maximum of polylogarithmic scaling at $\alpha=2$, and falls off on either sides, that is, $\alpha<2$ or $\alpha>2$, achieving the minimum values of $\Theta\left(1 /\left(n^{1 / 3} \sqrt{\log n}\right)\right)$ when $\alpha=0$, and $\Theta(1 / \sqrt{n \log n})$ when $\alpha$ goes to infinity (which corresponds to the scaling behavior of the traditional multi-hop wireless networks without DE links).

\section{SYNTHESIS OF RESULTS OF THEOREMS 4, 5 , AND 6}

Since Theorem 4 is obtained using Gupta and Kumar's methodology [1], and Theorems 5 and 6 are based on Theorems 2 and 3, we provide a synthesis between the capacity upper bound given in Theorems 4, and per-node throughput capacity given by Theorems 5 and 6 . There are three cases: 1) $\alpha=2$; 2) $0 \leq \alpha<2$; and 3) $\alpha>2$.

\section{A. The case of $\alpha=2$}

Using the normalization constant in (7) (in Appendix A), we can calculate $r_{2}$, which is the average distance of a DE link.

$$
\begin{aligned}
r_{2} & =\int_{r_{0}}^{\sqrt{\frac{n}{\pi}}} r f(r) 2 \pi r d r=\int_{r_{0}}^{\sqrt{\frac{n}{\pi}}} \frac{\Theta\left((\log n)^{-1}\right)}{r^{2}} 2 \pi r^{2} d r \\
& =\Theta\left(\frac{\sqrt{n}}{\log n}\right)
\end{aligned}
$$

Plugging $r_{2}$ into (2), we get

$$
\lambda \leq \frac{k_{2}}{\sqrt{n \log (n)}}+\frac{k_{3} p r_{2}}{\sqrt{n}}=\Theta\left((\log n)^{-1}\right)
$$

Thus, the achievable throughput obtained in Theorem 5 $\left((\log n)^{-3}\right)$ is within a polylogarithmic factor of the capacity upper bound given in (4).

\section{B. The case of $0 \leq \alpha<2$}

Using the normalization constant in (9) (in Appendix B), we have

$$
\begin{aligned}
r_{2} & =\int_{r_{0}}^{\sqrt{\frac{n}{\pi}}} r f(r) 2 \pi r d r=\int_{r_{0}}^{\sqrt{\frac{n}{\pi}}} \frac{n^{-(2-\alpha) / 2}}{r^{\alpha}} 2 \pi r^{2} d r \\
& =\Theta\left(n^{1 / 2}\right)
\end{aligned}
$$

Plugging $r_{2}$ into (2), we get

$$
\lambda \leq \frac{k_{2}}{\sqrt{n \log n}}+\frac{k_{3} p r_{2}}{\sqrt{n}}=\Theta(1)
$$

In this case, even though the necessary condition for CTL capacity scaling in Corollary 1 is satisfied $\left(r_{2}=\Theta\left(n^{1 / 2}\right)\right)$, and Theorem 4 provides capacity upper bound of $\Theta(1)$, the actual achievable capacity, as given in Theorem 6 , is much less.

\section{The case of $\alpha>2$}

Using the normalization constant in (13) (in Appendix B), we have

$$
\begin{array}{r}
r_{2}=\int_{r_{0}}^{\sqrt{\frac{n}{\pi}}} r f(r) 2 \pi r d r=\int_{r_{0}}^{\sqrt{\frac{n}{\pi}}} \frac{C}{r^{\alpha}} 2 \pi r^{2} d r \\
=\left\{\begin{array}{lc}
\Theta\left(n^{(3-\alpha) / 2}\right) & 2<\alpha \leq 3, \\
\Theta(1) & \alpha>3,
\end{array}\right.
\end{array}
$$

Plugging $r_{2}$ into Equation (2), we get

$$
\lambda \leq \frac{k_{2}}{\sqrt{n \log n}}+\frac{k_{3} p r_{2}}{\sqrt{n}}=\left\{\begin{array}{lr}
\Theta\left(n^{-(2-\alpha) / 2}\right) & 2<\alpha \leq 3, \\
\Theta\left(n^{-1 / 2}\right) & \alpha>3
\end{array}\right.
$$

Comparing the above upper bound with that given in Theorem 6 , which can be rewritten as $\Theta\left(n^{-(\alpha-2) /(2 \alpha-2)} / \sqrt{\log n}\right)=$ $\Theta\left(n^{-1 / 2+1 /(2 \alpha-2)} / \sqrt{\log n}\right)$, we can see that Theorem 4 actually provides a tighter upper bound than Theorem 6 .

We summarize the capacity upper bounds in Figure 2. Note that the CTL scaling is achieved only when $\alpha=2$, not with any other $\alpha$ values. One would imagine that smaller $\alpha$ values would favor longer DE links and thus provide higher capacity. That, however, does not happen. There are two reasons for this: 1) $r_{2}$ essentially saturates at $\alpha=2,\left(r_{2}=\Theta(\sqrt{n} / \log n)\right)$ for $\alpha=2$ and $r_{2}=\Theta\left(n^{1 / 2}\right)$ for $(0 \leq \alpha<2)$, so smaller $\alpha$ values do not bring much benefits in terms of the average span of a DE link; 2) as Kleinberg pointed out [19], since only local information is available, a DE link distribution with a stronger local bias $(\alpha=2)$ is better positioned to use such information than those with weaker local bias $(0<\alpha<2)$.

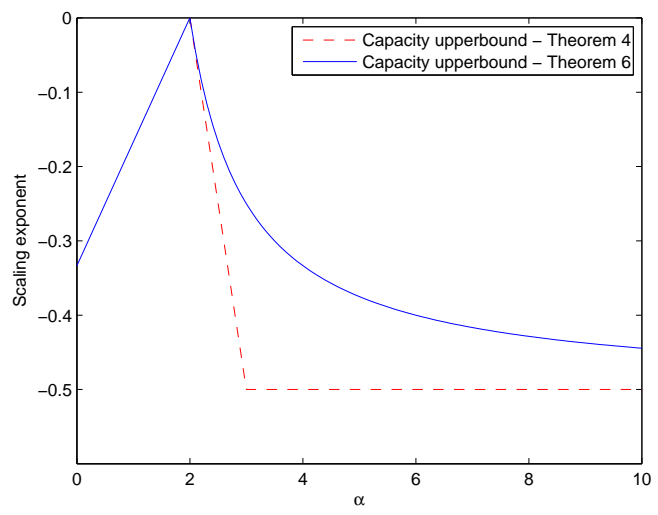

Fig. 2: Comparison of capacity upper bounds given by Theorem 4 (dashed line) and Theorem 6 (solid line).

\section{CONCLUSION}

In this paper, we provide an approach to CTL capacity scaling through the use of DE links. Our approach has none of the limitations of the previously proposed approaches. We provide the upper-bound on the capacity of the hybrid wireless networks that contain both DE and OD links. We show that when the probability distribution of DE links follows the inverse-square law, global routing information is not required to achieve CTL capacity scaling, which is impossible for any other power-law distributions. Our findings will benefit the practical design of large-scale wireless networks. 


\section{APPENDIX A}

\section{The Proof of Theorem 2}

We first compute the normalization constant $C$ of the probability distribution of the DE links. We consider a node at the center of the disc, which is similar to the treatment in [19], with the difference being the node is placed in the center of a square grid in [19]. Placing the node at another place makes the calculation complicated and ugly, but it does not change the scaling behavior with respect to $n$. The constant $C$ is derived by the normalization condition as follows

$$
\begin{gathered}
\int_{r_{0}}^{\sqrt{n / \pi}} f(r) 2 \pi r d r=\int_{r_{0}}^{\sqrt{n / \pi}} \frac{C}{r^{\alpha}} 2 \pi r d r=p \\
C=\frac{p}{\pi \log \left(\frac{n}{\pi r_{0}^{2}}\right)}=\Theta\left(\frac{1}{\log n}\right)
\end{gathered}
$$

In the above, the upper integration limit is set based on the fact that the area is $n$. The lower limit $r_{0}$ is introduced to avoid divergence. Since our model is a continuous approximation of a discrete network when $n$ is large, $r_{0}$ is on the order of the typical distance between two nearest neighbors, i.e., $r_{0}=\Theta(1)$.

Now we describe a communication scheme to achieve the hop counts prescribed by Theorem 2, using only local information. Let us fix a destination node and randomly select a source node, with high probability the distance between the source and the destination is $\Theta(\sqrt{n})$. The packet from the source reaches its destination in $O(\log n)$ phases. Each phase consists of $O(\log n)$ steps. In each step, the packet travels from one node to its neighboring node using an OD link. After arriving at a new node, the new node checks if it has a DE link that can reduce the current distance to the destination by half. If it has the DE link, the packet is sent through this link; otherwise the packet continues on to the next neighboring node using an OD link. In the last phase, the packet is within a distance of $O(1)$ to the destination node and can reach the destination node using a few OD links.

Since the diameter of the network is $\Theta(\sqrt{n})$, and each phase reduces the distance to the destination by a factor of 2 , so the packet can reach the destination in $O(\log n)$ phases.

The reason that each phase can be completed within $O(\log n)$ steps is as follows. Suppose the packet starts a phase at a node with a distance $d$ to the destination. We want to know the probability that the packet encounters a DE link whose other end node has a distance $d / 2$ to the destination in the subsequent steps. Since the events that nodes have DE links are independent, each step the packet takes is a Bernoulli trial. The event that the trial is successful is equivalent to that the current node has a DE link to any of the nodes in the disc $D$ of radius $d / 2$ centered at the destination, referring to Figure 3 . Such an event has a probability given by integrating the probability density over the area of $D$, i.e.,

$$
\begin{aligned}
P_{D E} & =\int_{D} \frac{C}{r^{2}} d A>\int_{D} \frac{C}{r_{\max }^{2}} d A=\frac{C|D|}{r_{\max }^{2}} \\
& =\frac{C \pi(d / 2)^{2}}{(3 d / 2)^{2}}=\Theta\left(\frac{1}{\log n}\right)
\end{aligned}
$$

Note that $r$ is the distance between the current node (not the destination) and an arbitrary node in $D$; and $r_{\max }$ is the maximum distance between the current node and any node in $D$, which occurs when there is a node sitting at the edge of $D$ that is diametrically opposite to the current node. The expected number of the Bernoulli trial until success is $1 / P_{D E}=O(\log n)$. Therefore the phase will be completed in $O(\log n)$ steps with high probability.

Since there are $O(\log n)$ phases, and each phase incurs $O(\log n)$ OD hops and 1 DE hop, we obtain the results in Theorem 2.

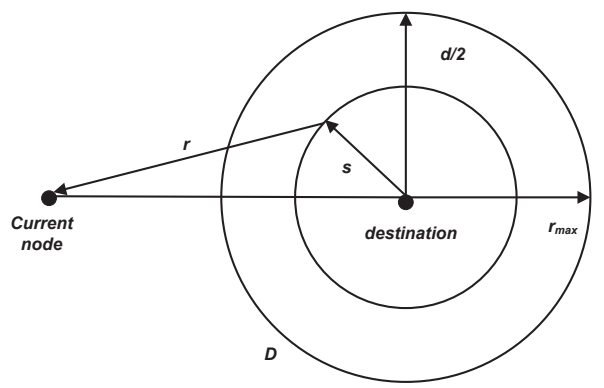

Fig. 3: The setup to compute the probability of encountering a DE link that brings the packet within the disc D

\section{APPENDIX B THE ProOF OF THEOREM 3}

The case of $0<\alpha<2$ : We place the node at the center of the disc. We discussed the reasoning for such placement in the first paragraph in the proof of Theorem 2. The normalization constant $C$ is derived by the following equations

$$
\begin{aligned}
\int_{r_{0}}^{\sqrt{n / \pi}} f(r) 2 \pi r d r & =\int_{r_{0}}^{\sqrt{n / \pi}} \frac{C}{r^{\alpha}} 2 \pi r d r=p \\
& =\frac{2 \pi C\left(\left(\frac{n}{\pi}\right)^{(2-\alpha) / 2}-r_{0}^{(2-\alpha)}\right)}{(2-\alpha)}=p \\
C & =\frac{(2-\alpha) p}{2 \pi}\left(\frac{\pi}{n}\right)^{(2-\alpha) / 2} \\
& =\Theta\left(\frac{1}{n^{(2-\alpha) / 2}}\right)
\end{aligned}
$$

In the above, we have omitted the term $r_{0}^{(2-\alpha)}$ in the second line since it is dominated by the term $(n / \pi)^{(2-\alpha) / 2}$ as $n$ goes to infinity.

Let $E$ denotes the event that the source node of a packet has a distance larger than $(n / 3 \pi)^{1 / 2}$ to the destination. Given the nodes are uniformly randomly distributed in the disc of area $n$, the probability of $\mathrm{E}$ occurring can be calculated as $\operatorname{Pr}[E]=1-\pi\left((n / 3 \pi)^{1 / 2}\right)^{2} / n=2 / 3$. Consider the disc $D$ of radius $n^{\beta}(\log n)^{1 / 4}$ centered at the destination, with the constant $\beta=(2-\alpha) / 6<1 / 3$. Thus, the source node is currently outside the disc when event $E$ occurs. We calculate the probability that the node has a DE link to a node within $D$ other than the destination by integrating the probability density 
over the area of $D$ as follows, refer to Figure 4.

$$
\begin{aligned}
P_{D E} & =\int_{D} \frac{C}{r^{\alpha}} d A<\int_{D} \frac{C}{r_{0}^{\alpha}} d A=\frac{C|D|}{r_{0}^{\alpha}} \\
& =\frac{C \pi\left(n^{\beta}(\log n)^{1 / 4}\right)^{2}}{r_{0}^{\alpha}} \\
& =\Theta\left(\frac{n^{2 \beta}(\log n)^{1 / 2}}{n^{(2-\alpha) / 2}}\right)=\Theta\left(\frac{\sqrt{\log n}}{n^{\beta}}\right)
\end{aligned}
$$

In the above, $r_{0}$ is the minimum distance between nodes introduced in the proof of Theorem 2, and we have used the equality $\alpha=(2-\alpha) / 6$.

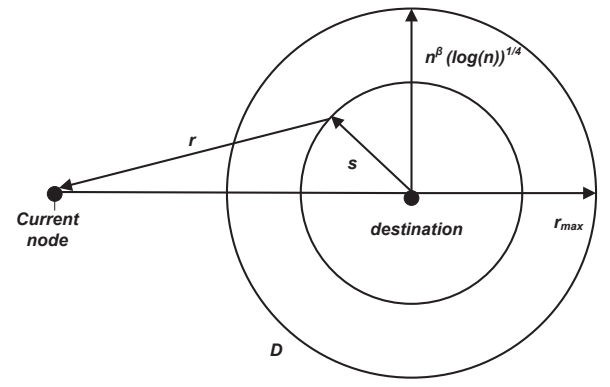

Fig. 4: The setup to compute the probability of encountering a DE link that brings the packet within the disc D

Let $F$ denotes the event that the packet jumps from the outside to the inside of the disc $D$ using $\delta / P_{D E}=\delta n^{\beta} / \sqrt{\log n}$ DE hops, where $\delta$ is a positive constant. Since each DE hop can be considered as an independent Bernoulli trial with the probability no more than $P_{D E}$, The probability of $F$ occurring can be made smaller than $1 / 3$ by appropriately choosing $\delta$. We can compute the joint probability of either $E$ not occurring or $F$ occurring as

$$
\operatorname{Pr}[\bar{E} \cup F] \leq \operatorname{Pr}[\bar{E}]+\operatorname{Pr}[F]=1 / 3+1 / 3=2 / 3
$$

For sufficiently large $n$, the joint probability of both $E$ occurring and $F$ not occurring is given by

$$
\operatorname{Pr}[E \cap \bar{F}]=1-\operatorname{Pr}[\bar{E} \cup F] \geq 1-2 / 3=1 / 3
$$

Let $G$ denotes the event that the packet can reach the destination within $\delta n^{\beta} / \sqrt{\log n}$ hops, in any OD and DE link combinations. We assert that $G$ can not happen if $E$ occurs but $F$ does not occur. We use the method of contradiction to prove the previous statement. Suppose $G$ happens. Given that each OD link covers a distance of $\sqrt{\log n}$, using $O\left(n^{\beta}\right)$ hops, it is impossible to travel the distance from the source to the destination, which is $(n / 3 \pi)^{1 / 2}$, using OD links alone. So, at least one hop must use a DE link. Further, the last time a DE link is used, the packet must jump inside the disc $D$ (otherwise using OD links alone is not enough to reach the destination), which contradict the assumption $F$ does not occur. Therefore we have

$$
\operatorname{Pr}[G \mid E \cap \bar{F}]=0
$$

Let $h$ be the random variable indicating the number of hops traveled by the packet to reach the destination, as a consequence of Equation (12), we have

$$
E[h \mid E \cap \bar{F}]>\delta n^{\beta} / \sqrt{\log n}
$$

Finally, we have

$$
E[h]>E[h \mid E \cap \bar{F}] \operatorname{Pr}[E \cap \bar{F}]>\frac{1}{3} \delta n^{\beta} / \sqrt{\log n}
$$

which is the result of the first part of the theorem.

The case of $\alpha>2$ : The normalization constant $C$ is derived by the following equations

$$
\begin{aligned}
\int_{r_{0}}^{\sqrt{n / \pi}} f(r) 2 \pi r d r & =\int_{r_{0}}^{\sqrt{n / \pi}} \frac{C}{r^{\alpha}} 2 \pi r d r=p \\
& =\frac{2 \pi C\left(r_{0}^{(2-\alpha)}-\left(\frac{n}{\pi}\right)^{(2-\alpha) / 2}\right)}{(\alpha-2)}=p \\
C & =\frac{(\alpha-2) p}{2 \pi} r_{0}^{(\alpha-2)}
\end{aligned}
$$

In the above, we have omitted the term $(n / \pi)^{(2-\alpha) / 2}$ in the second line since it is dominated by the term $r_{0}^{(2-\alpha)}$ as $n$ goes to infinity.

Again, we denote $E$ as the event that the source node of a packet has a distance larger than $(n / 3 \pi)^{1 / 2}$ to the destination, which has the probability $\operatorname{Pr}[E]=2 / 3$. Let $\beta^{\prime}=(\alpha-2) /(2 \alpha-2), \gamma=1 /(2 \alpha-2)$. We calculate the probability that a packet has a DE link that covers a distance larger than $n^{\gamma}$ as

$$
P_{n, \gamma}=p-\int_{r_{0}}^{n^{r}} \frac{C}{r^{\alpha}} 2 \pi r d r=\frac{p r_{0}^{\alpha-2}}{n^{\gamma(\alpha-2)}}=\Theta\left(n^{-\beta^{\prime}}\right)
$$

We denote $F^{\prime}$ as the event that the packet encounters a DE link that covers a distance larger than $n^{\gamma}$ in $\delta^{\prime} n^{\beta^{\prime}}$ hops, where $\delta^{\prime}$ is a positive constant satisfying $\delta^{\prime}<(3 \pi)^{-1 / 2}$. Again, each hop can be considered as an independent Bernoulli trial with the probability of $P_{n, \gamma}$. By choosing a sufficient small value for $\gamma^{\prime}$, we can make the probability of $F^{\prime}$ occurring no larger than $1 / 3$. We can compute the joint probability of either E not occurring or $F^{\prime}$ occurring as

$$
\operatorname{Pr}\left[\bar{E} \cup F^{\prime}\right] \leq \operatorname{Pr}[\bar{E}]+\operatorname{Pr}\left[F^{\prime}\right]=1 / 3+1 / 3=2 / 3
$$

The joint probability of both E occurring and F not occurring is given by

$$
\operatorname{Pr}\left[E \cap \overline{F^{\prime}}\right]=1-\operatorname{Pr}\left[\bar{E} \cup F^{\prime}\right] \geq 1-2 / 3=1 / 3
$$

We denote $G^{\prime}$ as the event that the packet can reach the destination within $\delta^{\prime} n^{\beta^{\prime}}$ hops. We assert that $G^{\prime}$ can not happen if $E$ occurs but $F^{\prime}$ does not occur. Suppose $G^{\prime}$ happens. Since $F^{\prime}$ does not occur, each hop covers a distance no larger than $n^{\gamma}$. Then, the maximum distance covered by the packet in $\delta^{\prime} n^{\beta^{\prime}}$ hops is given by

$$
\delta^{\prime} n^{\beta^{\prime}} n^{r}=\delta^{\prime} n^{1 / 2}<\left(\frac{n}{3 \pi}\right)^{1 / 2}
$$

Therefore, the packet can not reach the destination (a contradiction). So we have

$$
\operatorname{Pr}\left[G \mid E \cap \overline{F^{\prime}}\right]=0
$$

Let $h^{\prime}$ be the random variable indicating the number of hops traveled by the packet to reach the destination, as a consequence of Equation (16), we have

$$
E\left[h^{\prime} \mid E \cap \overline{F^{\prime}}\right]>\delta^{\prime} n^{\beta^{\prime}}
$$


Finally, we have

$$
E\left[h^{\prime}\right]>E\left[h^{\prime} \mid E \cap \overline{F^{\prime}}\right] \operatorname{Pr}\left[E \cap \overline{F^{\prime}}\right]>\frac{1}{3} \delta^{\prime} n^{\beta^{\prime}}
$$

Now suppose $\gamma=0$, then $\alpha=\infty$ and $\beta^{\prime}=1 / 2$. In such case an OD hop covers a larger distance $\sqrt{\log n}$ than $n^{\gamma}=1$. It takes $O\left(n^{\beta^{\prime}} / \sqrt{\log n}\right)=O(\sqrt{n / \log n})$ hops to reach the destination that has a distance of $(n / 3 \pi)^{1 / 2}$.

\section{ACKNOWLEDGMENT}

This research is funded in part by NSF grant CNS-1248109. The information here does not reflect the position or the policy of the federal government.

\section{REFERENCES}

[1] P. Gupta and P. R. Kumar, "The capacity of wireless networks," IEEE Trans. Inf. Theory, vol. 42, pp. 388-404, Mar. 2000.

[2] A. Jovicic, P. Viswanath, and S. Kulkarni, "Upper bounds to transport capacity of wireless networks," IEEE Trans. Inf. Theory, vol. 50, no. 11, pp. 2555-2565, Nov. 2004.

[3] L.-L. Xie and P. Kumar, "A network information theory for wireless communications: Scaling laws and optimal operation," IEEE Trans. Inf. Theory, vol. 50, no. 5, pp. 748-767, May 2004.

[4] O. Lévêque and E. Telatar, "Information theoretic upper bounds on the capacity of large, extended ad hoc wireless networks," IEEE Trans. Inf. Theory, vol. 51, no. 3, pp. 858-865, Mar. 2005.

[5] F. Xue, L. Xie, P. Kumar, "The transport capacity of wireless networks over fading channels," IEEE Trans. Inf. Theory, vol. 51, no. 3, pp. 834 847, March 2005.

[6] L.-L. Xie and P. Kumar, "On the path-loss attenuation regime for positive cost and linear scaling of transport capacity in wireless networks," IEEE Trans. Inf. Theory, vol. 52, no. 6, pp. 2313-2328, Jun. 2006.

[7] A. Özgür, O. Lévêque and E. Preissmann, "Scaling laws for one and twodimensional random wireless networks in the low attenuation regime," IEEE Trans. Inf. Theory, vol. 53, no. 10, pp. 3573-3585, Oct. 2006.

[8] S. Aeron and V. Saligrama, "Wireless ad hoc networks: Strategies and scaling laws for the fixed SNR regime," IEEE Trans. Inf. Theory, vol. 53, no. 6, pp. 2044-2059, Jun. 2007.

[9] M. Franceschetti, O. Dousse, D. Tse and P. Thiran, "Closing the gap in the capacity of wireless networks via percolation theory," IEEE Trans. Inf. Theory, vol. 53, no. 3, pp. 1009-1018, Mar. 2007

[10] A. Özgür, R. Johari, D. Tse and O. Lévêque, "Information-theoretic operating regimes of large wireless networks," IEEE Trans. Inf. Theory, vol. 56, no. 1, pp. 427-437, January 2010.

[11] M. Grossglauser and D. Tse, "Mobility increases the capacity of wireless ad hoc networks," in Proc. of INFOCOM, 2001.

[12] A. Özgür, O. Lévêque and D. Tse, "Hierarchical cooperation achieves optimal capacity scaling in ad hoc networks," IEEE Trans. Inf. Theory, vol 53, no. 10, pp. 3549-3572, October 2007.

[13] A. Özgür, O. Lévêque, and D. Tse, "Linear capacity scaling in wireless networks: beyond physical limits?" in Proc. of ITA Workshop, 2010.

[14] J. Li, C. Blake, D. De Couto and H. I. Lee and R. Morris, "Capacity of ad hoc wireless networks," in Proc. of ACM MobiCom, 2001.

[15] B. Liu, P. Thiran and D. Towsley, "Capacity of a wireless ad hoc network with infrastructure," in Proc. of ACM MobiHoc, 2007.

[16] S. Yi, Y. Pei, and S. Kalyanaraman, "On the capacity improvement of ad hoc wireless networks using directional antennas," in Proc. of ACM MobiHoc, 2003.

[17] D. Wang and A. Abouzeid, "Throughput capacity of hybrid radiofrequency and free-space-optical (rf/fso) multi-hop networks," in Proc. of ITA Workshop, 2007.

[18] S. Das, et al., "Requirements and challenges for tactical free-space lasercomm," in Proc. of IEEE MILCOM, 2008.

[19] J. Kleinberg, "The small-world phenomenon: An algorithmic perspective," in Proc. of ACM Symposium on Theory of Computing, 2000.

[20] J. Kleinberg, "Complex networks and decentralized search algorithms," in Proc. of the International Congress of Mathematicians (ICM), 2006.

[21] FCC Report and Order 03-248, "Allocations and Service Rules for the 71-76 GHz, 81-86 GHz, and 92-95 GHz Bands," November, 2003.

[22] I. Stojmenovic, "Position based routing in ad hoc networks," IEEE Communications Magazine, 40 (7), 2002.
[23] C. Peraki and S. D. Servetto, "On the Maximum Stable Throughput Problem in Random Networks with Directional Antennas," in Proc. of ACM MobiHoc, 2003.

[24] S. Milgram, "The small world problem," Psychology Today, 1(61), 1967.

[25] D. Watts and S. Strogatz, "Collective dynamics of small-world networks," Nature, vol. 393, no. 440, 1998.

[26] P. Erdös, A. Rényi, "On Random Graphs. I,” Publicationes Mathematicae, vol. 6, pp. 290-297, 1959.

[27] H. Huang, Y. Jaradat, R. Asorey-Cacheda and S. Misra, "Towards achieving linear capacity scaling in wireless networks through directed energy links," Tech Report, Klipsch School of Electrical and Computer Engineering, New Mexico State University, available at: http://ece.nmsu.edu/ hhuang/TR-Capacity-Linear.pdf

[28] T. Wang, L. Song and Z. Han, "Collaborative Data Dissemination in Cognitive VANETs Networks with Sensing-Throughput Tradeoff," in Proc. of First IEEE International Conference on Communications in China (ICCC), 2012 (Invited Paper).

[29] G. Zhang, Y. Xu, X. Wang and M. Guizani, "Capacity of Hybrid Wireless Networks with Directional Antenna and Delay Constraint," IEEE Transactions on Communications, vol. 58, no. 7, pp. 2097-2106, 2010.

[30] Y. Zhang, L. Song, W. Saad, Z. Dawy and Z. Han, 'Exploring Social Ties for Enhanced Device-to-Device Communications in Wireless Networks," in Proc. of IEEE Globe Communication Conference (Globecom), 2013.

[31] G. Zhang, Y. Xu, X. Wang, X. Tian, J. Liu, X. Gan, H. Yu and L. Qian: "Multicast Capacity for VANETs with Directional Antenna and Delay Constraint," IEEE Journal on Selected Areas in Communications, vol. 30, no. 4, pp. 818-833, 2012.

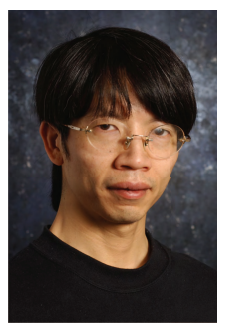

Hong Huang received his B.E. degree from Tsinghua University, Beijing, China, and M.S. and Ph.D. degrees from Georgia Institute of Technology in 2000 and 2002, respectively, all in electrical engineering. He is currently an associate professor with the Klipsch School of Electrical and Computer Engineering at the New Mexico State University. His current research interests include wireless sensor networks, mobile ad hoc networks, network security, and optical networks. He is a member of the IEEE.

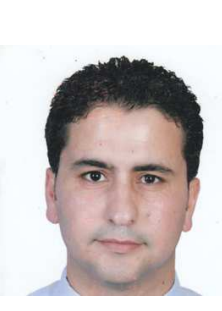

Yousef Jaradat received his B.S. in electrical and computer engineering from Jordan University of Science and Technology, Irbid, Jordan, in 2000, M.S in computer science from Amman Arabia University, Amman, Jordan, in 2007, and his Ph.D. in electrical and computer engineering from New Mexico State University, Las Cruces, New Mexico, USA, in 2012. His research includes work in the capacity of wireless networks, Obstacles analysis in the network area, and simulation of wireless networks.

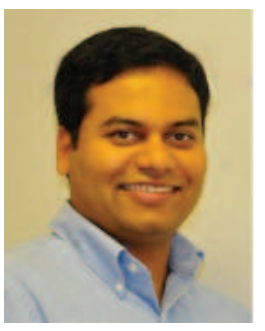

Satyajayant Misra (SM'05, M'09) is an assistant professor in computer science at New Mexico State University. He completed his M.Sc. in Physics and Information Systems from BITS, Pilani, India in 2003 and his Ph.D. in Computer Science from Arizona State University, Tempe, AZ, USA, in 2009. His research interests include wireless networks and the Internet, supercomputing, and smart grid architectures and protocols. He serves on several IEEE journal editorial boards and conference executive committees (Communications on Surveys and Tutorials, Wireless Communications Magazine, SECON 2010, INFOCOM 2012). He has authored more than 36 peer-reviewed IEEE/ACM journal articles and conference proceedings. More information can be obtained at www.cs.nmsu.edu/ misra.

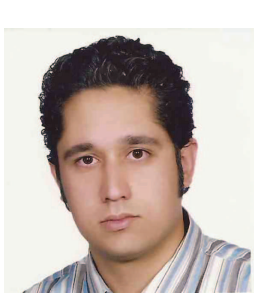

Reza Tourani received his B.S. in computer engineering from IAUT, Tehran, Iran, in 2008, and M.S. in computer science from New Mexico State University, Las Cruces, NM, USA, in 2012. From 2013, he started his Ph. D. at New Mexico State University. His research interests include Wireless Protocols Design and Optimizations, Future Internet Architecture, and Privacy and Security in Wireless Networks. 